\title{
Posterior Reversible Encephalopathy Syndrome Caused by Autonomic Dysreflexia in an Adolescent after Spinal Cord Injury
}

\author{
Javid Sardarzada ${ }^{*}$, Sibel Öz ${ }^{1}$, Dilara Mat ${ }^{2}$, Ibrahim Öncel ${ }^{1}$, Ali Düzova ${ }^{3}$ and Banu Anlar ${ }^{1}$ \\ ${ }^{1}$ Faculty of Medicine, Division of Pediatric Neurology, Hacettepe University, Ankara, Turkey \\ ${ }^{2}$ Faculty of Medicine, Department of Pediatrics, Hacettepe University, Ankara, Turkey \\ ${ }^{3}$ Faculty of Medicine, Division of Pediatric Nephrology, Hacettepe University, Ankara, Turkey
}

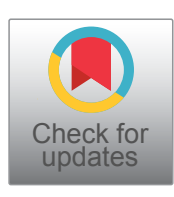

\begin{abstract}
The posterior reversible encephalopathy syndrome (PRES) is a clinico-radiological entity caused by impairment of cerebral autoregulation leading to vasogenic edema. Common etiologies include immunosuppressive drugs, autoimmune disorders and hypertension.

We report a 17-year-old tetra paretic patient who presented with PRES to the emergency room five months after having suffered a spinal cord injury. The etiology of PRES was confirmed as autonomic dysreflexia causing hitherto unrecognized brief hypertensive episodes.

This is the first report of the association of spinal cord injury, autonomic dysreflexia and PRES in pediatric age group. The present case draws attention to short episodes of hypertension that initially go unnoticed but may progress to encephalopathy in patients with spinal cord injury. Close blood pressure monitorization and a detailed history of precipitating factors allowed etiological diagnosis and appropriate treatment in our patient.
\end{abstract}

\section{Keywords}

Autonomic dysreflexia, Sympathetic, Spinal cord injury, Posterior reversible encephalopathy syndrome, Blood pressure, Hypertension.

\section{Introduction}

The posterior reversible encephalopathy syndrome (PRES) typically presents with headache, altered consciousness, visual disturbances and seizures. Brain imaging studies show vasogenicedema and vascular leakage resulting from impaired autoregulation of cerebral perfusion and endothelial dysfunction [1,2]. The most frequent etiology of PRES in children is acute hypertension followed by autoimmune diseases and immunosuppressive drugs [3]. Secondary causes of hypertension including renovascular diseases, pheochromocytoma, ganglioneuroma, primary aldosteronism, acute/chronic kidney disease, hyperthyroidism and use of recreational drugs (e.g., amphetamine, cocaine) are among the main reasons of PRES in children and adolescents [4]. Acute elevations or fluctuations in blood pressure (BP) may be more influential in the development of PRES than sustained hypertension $[5,6]$.

Here we present an unusual etiology of hypertension, autonomic dysreflexia, resulting in PRES in an adolescent.

\section{Case Report}

A 17-year-old male experienced headache that lasted 2 hours followed by vision loss, a tonic-clonic seizure and inability to speak for 4 minutes.
His past medical history was remarkable for an acute spinal cord injury $(\mathrm{SCl})$ after diving into a shallow pool 5 months ago. He had developed tetra paresis and partial sensory disturbance below spinal level C5. A non-displaced midline vertical fracture line in $\mathrm{C} 5-\mathrm{C} 6$ vertebrae and another one extending to transverse processes and laminae at $\mathrm{C} 6$ were observed. After a vertebral fusion operation of C5-C6, he had been discharged with bed rest, low-molecular-weight heparin prophylaxis, physiotherapy and intermittent urethral catheterization.

Upon presentation to the emergency room, his BP was $110 / 70 \mathrm{mmHg}$, heart rate 60 b.p.m, and temperature, 36

*Corresponding author: Javid Sardarzada, Faculty of Medicine, Division of Pediatric Neurology, Hacettepe University, Ankara, Turkey, Tel: +905452887606

Accepted: August 07, 2021

Published online: August 09, 2021

Citation: Sardarzada J, Öz S, Mat D, et al. (2021) Posterior Reversible Encephalopathy Syndrome Caused by Autonomic Dysreflexia in an Adolescent after Spinal Cord Injury. J Pediatr Neurol Neurosci 5(2):142-144 


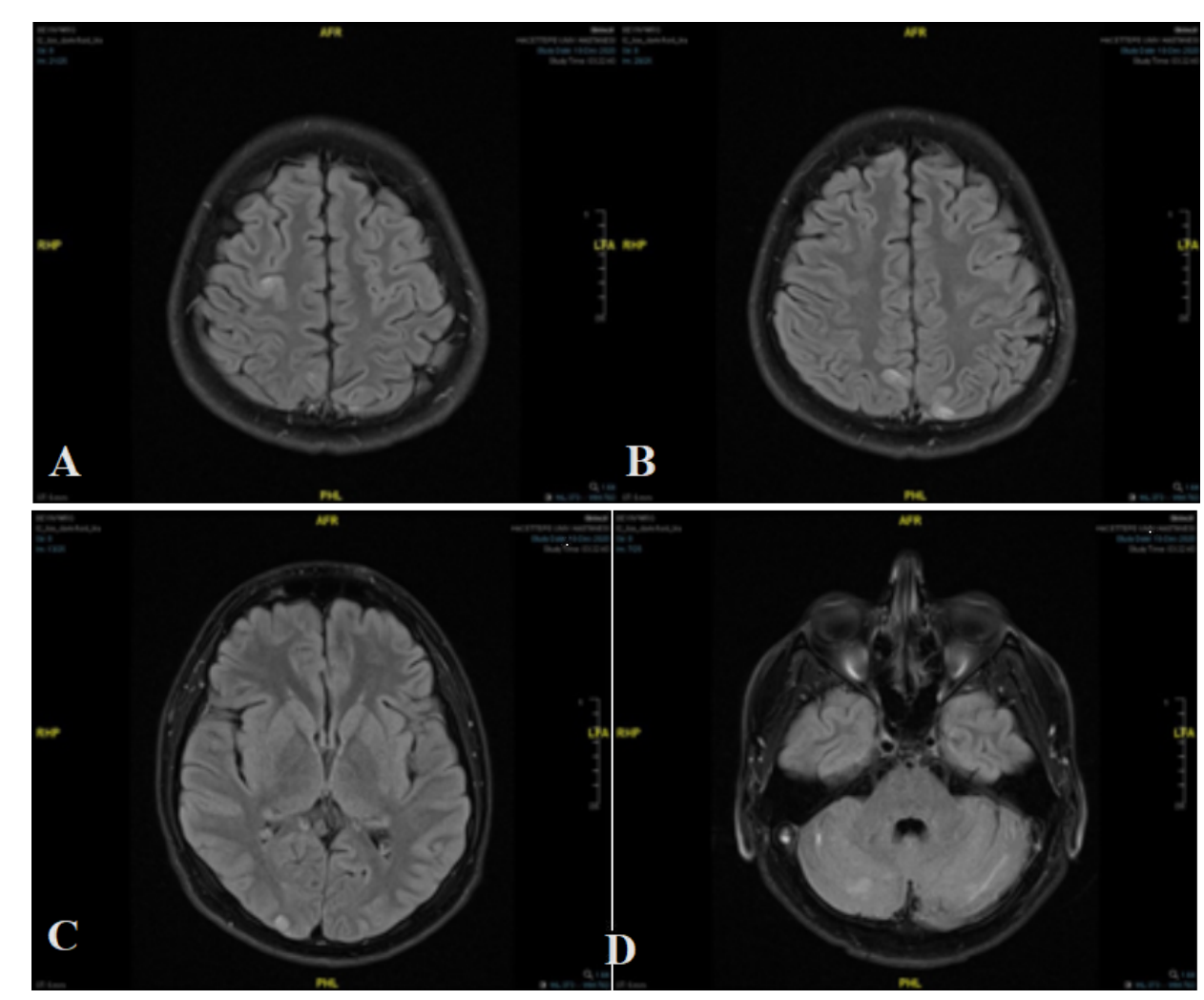

Figure 1: Fluid-attenuated in version recovery (FLAIR) sequencesshowing (arrows) multi focal cortical hyper intensities anterior to the right precentralgyrus A) bilateralparietooccipitalareas; $B, C$ ) and right cerebellar hemisp here; D) on brain MRI.

${ }^{\circ} \mathrm{C}$. His consciousness and vision were normal. He had tetra paresis with little muscle contraction in lower, and limited movement in upper extremities. Brain MRI showed multifocal, T2- and FLAIR hyper intense cortical lesions scattered in the right cerebellar hemisphere, anterior to the right precentral gyrus, and bilateral parietal and occipital areas. Some of these lesions showed facilitated diffusion while others had focal limited diffusion suggesting PRES (Figure 1).

The patient was admitted for etiological investigation and management. During the first day, hourly BP measurements revealed normal results (systolic BP ranged between 90-110 $\mathrm{mmHg}$ and diastolic BP ranged between $60-70 \mathrm{mmHg}$ ); but continuous monitorization revealed several hypertensive recordings up to $140 / 100 \mathrm{mmHg}$ lasting 5-10 minutes every few days, usually associated with forced bowel movements of the patient: BP was starting to raise with abdominal discomfort and returned to normal after defecation. Further history-taking revealed that the patient had similar discomfort episodes after cessation of laxative (lactulose, bisacodyl) use which had caused diarrhea. Investigation for hypertension including renal function tests, abdominal and cardiac ultrasonography, Doppler ultrasonography of the renal arteries and electrocardiogram, returned all normal. Urine culture revealed $\mathrm{E}$. coli $\left(10^{5} \mathrm{cfu} / \mathrm{ml}\right)$ which was treated with antibiotics. The episodic hypertension was ultimately attributed to autonomic dysfunction (AD). Management was initiated with non-pharmacological measures including dietary recommendations for constipation, raising the head of his bed and avoiding triggering stimuli; laxatives (macrogol or sennosids) were used as needed. After observation of normal BP for one week, the patient was discharged with home rehabilitation program. During the follow-up home $\mathrm{BP}$ monitoring and $\mathrm{BP}$ at regular visits remained normal; the patient had no new episodes of PRES.

\section{Discussion}

Autonomic dysfunction was initially described by Guttmann and White ridge in 1947 who observed that bladder distention in patients with $\mathrm{SCl}$ was accompanied by hypertension, bradycardia, and diaphoresis above the level of the injury [7]. Cutaneous or visceral stimulation below the level of the $\mathrm{SCl}$ initiates afferent impulses that elicit reflex sympathetic activity. The latter leads to diffuse vasoconstriction, typically in the lower parts of the body, and a rise in blood pressure [8]. Stimulation of the lower urinary tract is the most common recognized precipitant of $A D$, but other stimuli to be considered are rectal distension, faecal impaction, painful stimuli due to ingrown toenails or fractures, and upper and lower gastrointestinal tract diseases [8-10]. Up to $90 \%$ of patients with cervical or high thoracic $\mathrm{SCl}$ above $\mathrm{T} 6$ are susceptible to $A D$, and higher levels carry greater risk [8]. PRES associated with $A D$ has only recently been described in reports of 18-55 year old patients with $\mathrm{SCl}$ between $\mathrm{C2}$ T4 [7]. Some had associated medical factors like fever, assisted cough maneuvers, urinary tract infection, or sepsis. They presented symptoms of headache, visual disturbance, and seizures associated with BP ranging between normal to 188/96 mm Hg [9]. 
Citation: Sardarzada J, Öz S, Mat D, et al. (2021) Posterior Reversible Encephalopathy Syndrome Caused by Autonomic Dysreflexia in an Adolescent after Spinal Cord Injury. J Pediatr Neurol Neurosci 5(2):142-144

The association of PRES and $\mathrm{SCl}$ with $\mathrm{AD}$ is not well known in the pediatric neurology literature. The presenting symptoms of this patient initially suggested an ischemic cerebral event resulting from cerebral thromboembolism due to immobility. The MRI revealed the unexpected findings of PRES. Although the etiology of PRES commonly includes hypertension and rapid elevations in blood pressure, many patients do not have sustained hypertension: brief $\mathrm{BP}$ elevations or fluctuations may be more influential particularly in patients with low/normal baseline BP and impaired cerebral vascular autoregulation [5]. Our patient's rapid BP changes were only noticed during inpatient follow-up. After exclusion of common etiologies, his hypertensive episodes were attributed to $A D$ due to $\mathrm{SCl}$.

This case had several teaching points. The patient being fully conscious at presentation, PRES was not suspected clinically. Hypertension was specifically considered for PRES, and only demonstrated under continuous BP monitorization. The symptoms of abdominal discomfort and constipation had not been given at admission medical history although they were significant in their association with BP changes. Therefore, this report illustrates daily and common causes of discomfort might be underestimated in $\mathrm{SCl}$ patients, as well as brief episodic rises in BP. Clinicians, patients and caregivers of $\mathrm{SCl}$ patients should be aware of $\mathrm{AD}$ for early diagnosis and treatment. Although long term outcome of $A D$ and associated PRES are unclear, early recognition and timely intervention are likely to reduce these episodes leading to hospitalization and extensive diagnostic investigations.

\section{Informed Consent}

Informed consent was obtained from the patient for the case report.

\section{Acknowledgements}

None.

\section{Funding}

There are no sources of financial support for the work reported in this paper.

\section{Declaration of Interest}

Authors have no conflict of interest to declare.

\section{References}

1. Hinchey J, Chaves C, Appignani B, et al. (1996) A reversible posterior leukoencephalopathy syndrome. N Engl J Med 334: 494-500.

2. Fugate JE, Rabinstein AA (2015) Posterior reversible encephalopathy syndrome: clinical and radiological manifestations, pathophysiology and outstanding questions. Lancet Neurol 14: 914-925.

3. Cordelli DM, Marra C, Ciampoli L, et al. (2021) Posterior Reversible Encephalopathy Syndrome in infants and young children. Eur J Paediatr Neurol 30: 128-333.

4. Chen TH (2020) Childhood posterior reversible encephalopathy syndrome: Clinicoradiological characteristics, managements and outcome. Front Pediatr 11: 585

5. Rabinstein AA, Mandrekar J, Merrell R, et al. (2012) Blood pressure fluctuations in posterior reversible encephalopathy syndrome. J Stroke Cerebrovasc 21: 254-258.

6. Zimering JH, Mesfin A (2018) Posterior reversible encephalopathy syndrome following elevated mean arterial pressures for cervical spinal cord injury. J Spinal Cord Med 41: 111-114.

7. Hubbard ME, Phillips AA, Charbonneau R, et al. (2019) PRES secondary to autonomic dysreflexia: A case series and review of the literature. J Spinal Cord Med 29: 1-7.

8. Allen KJ, Leslie SW (2021) Autonomic dysreflexia. StatPearls. StatPearls.

9. Matias AC, Rocha J, Cerqueira ME, et al. (2013) Autonomic dysreflexia and posterior reversible encephalopathy syndrome. Am J Phys Med Rehabil 92: 453-458.

10. KöseoğluE, Karataş GK (2011) Autonomic Dysreflexia in Spinal Cord Injury. J PMR Sci 14: 57-62.

DOI: $10.36959 / 595 / 426$

Copyright: (c) 2021 Sardarzada J. This is an open-access article distributed under the terms of the Creative Commons Attribution License, which permits unrestricted use, distribution, and reproduction in any medium, provided the original author and source are credited. 